Synchrotron Radiation Following the Impact of P/Comet Shoemaker-levy 9", submitted to Science.

References:

- de Pater, I., and M.J. Klein, 1989, in Time Variable Phenomena in the Jovian System, M.S. Belton et al. (eds) p. 139-150.

- Klein, M.J., T.J. Thompson, and S. Bolton, 1989, in Time-variable phenomena in the Jovian system, M.S. Belton et al. (eds), NASA SP-494, 151-155.

\title{
REVIEW OF THE CALAR ALTO COMET CAMPAIGN
}

T.M. Herbst (MPI Astron., Heidelberg), D.P. Hamilton (MPI Kernphysik, Heidelberg), H. Böhnhardt (U. Sternewarte, München), J.L. Ortiz-Moreno (Inst. Astrofis. Andalucia, Granada), A. Richichi (MPI Astron., Heidelberg and Os. Astrofis. Andalucia, Granada), K. Birkle (MPI Astron., Heidelberg), G. Calamai (Os. Astrofis. Arcetri, Firenze), A. Fiedler, K.-H. Mantel (U. Sternewarte, München), B. Stecklum (MPG Res. Group, Jena), U. Thiele (MPI Astron., Heidelberg), P.D. Nicholson (Cornell U., Ithaca), S.J. Kim (U. Maryland)

A collaboration of more than a dozen astronomers from seven different countries came together to observe the ten fragment impacts visible from the Calar Alto Observatory in Spain. The weather was excellent, and the team acquired data on each of the A, E, H, L, P2, Q2, Q1, S, T, and U impacts.

The observers at the $3.5 \mathrm{~m}$ telescope (Herbst, Hamilton, Böhnhardt, Ortiz-Moreno) conducted an intense program of near infrared imaging and spectroscopy using the MAGIC infrared camera. MAGIC contains a $256 \times 256$ $\mathrm{HgCdTe}$ detector array and is sensitive between 1 and $2.5 \mu \mathrm{m}$. All impacts were detected, except P2, T, and U. Light curves in the $2.3 \mu \mathrm{m}$ absorption band of $\mathrm{CH}_{4}$ show precursor flashes for the A (see figure 1), H, L, Q2, and Q1 impacts. In the case of Q2, the precursor was comparable in brightness to the main peak of emission 7 minutes later, and in the case of the $H$ and $L$ impacts, careful reduction of the data after the IAU colloquium showed that the precursors were double. Hamilton et al. discuss the nature of these precursors in a companion abstract in this volume. The MAGIC camera has motorized slits and grisms, allowing the team to switch to spectroscopy mode within $\sim 30$ seconds. Spectral sequences taken during the main brightness peaks of the $\mathrm{H}$ and $\mathrm{L}$ impacts show strong emission features from the $\Delta v=2$ bandhead emission of CO. These bandheads indicate the presence of hot $\sim 2000 \mathrm{~K}$ molecular 
gas. The $\mathrm{CO}$ emission faded and disappeared within 10 minutes of maximum light, suggesting that this hot phase is short-lived.

Two different observing teams shared time on the $2.2 \mathrm{~m}$ telescope. The MPIA/Arcetri group (Richichi, Calamai) used a fast infrared photometer to measure the $H$ and $Q$ lightcurves in a $3.1 \mu \mathrm{m}$ narrow band filter. The curves, with a time resolution of $\sim 1 \mathrm{sec}$, appear in figure 2. Note the different tails of the main peaks. An attempt to detect the flash of the A impact on Europa at $2.2 \mu \mathrm{m}$ produced an upper limit 6-7 mag fainter than the satellite. The München team (Mantel, Fiedler) used the MEKASPEK instrument to observe night-time impacts. MEKASPEK is a high speed $(50 \mathrm{~Hz})$, multiaperture, fiber-fed spectrophotometer with $\frac{\lambda}{\Delta \lambda} \sim 50$. Four fibers allowed simultaneous monitoring of the impact site, any visible satellites, and the adjacent sky. A search for reflected impact flashes on Europa during the $L$ event with 0.5 second time resolution showed no detectable signal in the initial analysis. More thorough reduction is underway.

At the $1.2 \mathrm{~m}$ telescope, a fourth team (Thiele, Birkle) imaged the planet at visible wavelengths using a CCD detector. Their images showed dark spots corresponding to the impact sites. None of the events produced a detectable flash.

Coordination and communication between all three telescopes proved invaluable in tracking and identifying these dynamic events. The MAGIC team continued to monitor the structure and evolution of the impact sites for approximately a week after the last fragment struck the planet, (and intermittently for the subsequent two months).

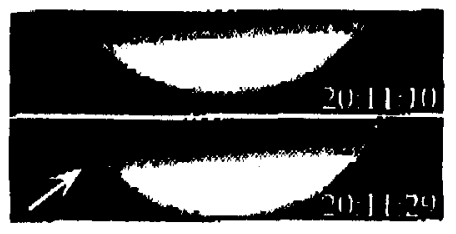

Fig.1 Impact $\triangle$ Precursor 16 July 1994, 20:11:29 UT

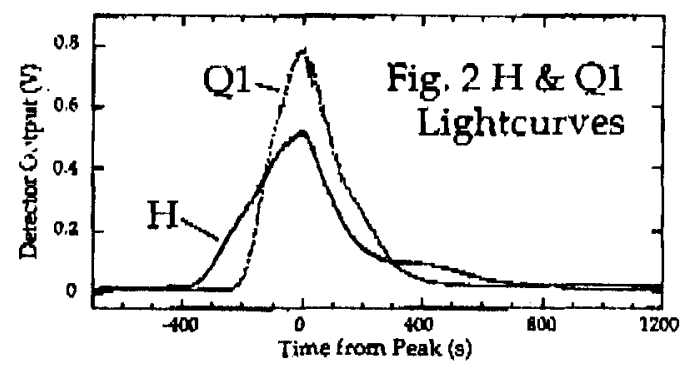

Figure: The precursor of the $\mathbf{A}$ impact (left), and a comparison of the $H$ and $Q$ lightcurves (right) 